\title{
室温下叔丁醇钾促进烷基芳基硫醚的合成与机理研究
}

\author{
郭芳杰何雨轩王景芸孙京* 周明东* \\ (辽宁石油化工大学化学与材料科学学院 抚顺 113001)
}

\begin{abstract}
摘要 研究了在无金属参与条件下芳基溴化物与烷基硫醇的 $\mathrm{C}-\mathrm{S}$ 交叉偶联反应. 研究表明在室温、 $\mathrm{KO}^{t} \mathrm{Bu}$ 强碱作用 条件下，各种缺电子的芳基溴化物与烷基硫醇均可以顺利地发生 $\mathrm{C}-\mathrm{S}$ 交叉偶联反应，高产率地得到了一系列烷基芳基 硫醚类产物. 本研究同时对非金属参与的偶联反应机理进行了探讨.

关键词 $\mathrm{C}-\mathrm{S}$ 交叉偶联; 芳基溴化物; 机理；烷基芳基硫醚
\end{abstract}

\section{Potassium tert-Butoxide Promoted Formation of Alkyl Aryl Thioethers at Room Temperature: Synthesis and Mechanism}

\author{
Guo, Fangjie He, Yuxuan Wang, Jingyun Sun, Jing* Zhou, Mingdong* \\ (School of Chemistry and Materials Science, Liaoning Shihua University, Fushun 113001)
}

\begin{abstract}
The $\mathrm{C}-\mathrm{S}$ cross-coupling of aryl halides with alkyl thiols under transition metal-free conditions was investigated Good to excellent yields can be obtained for a variety of electron-poor aryl halides with alkyl thiols in the presence of $\mathrm{KO}^{t} \mathrm{Bu}$ even at room temperature. The mechanisms for transition metal-free coupling reactions are discussed.
\end{abstract}

Keywords $\mathrm{C}-\mathrm{S}$ cross-coupling; aryl halides; mechanism; alkyl aryl thioethers

Aryl sulfides are important synthetic intermediates which can be used in biological or pharmaceutical industry. ${ }^{[1]}$ Catalytic cross-coupling of aryl halides with thiols proved to be one of very effective method for the construction of $\mathrm{C}-\mathrm{S}$ bond, wherein palladium ${ }^{[2]}$ and copper $^{[3]}$ salts or their complexes have been commonly used as effective catalysts under strong basic conditions. It is generally believed that the transition metal catalyzed coupling of aryl halides with thiols firstly undergoes the oxidative addition of $\mathrm{M}(0)$ with $\mathrm{ArX}$, then the ligand exchanges of $\mathrm{Ar}-\mathrm{M}-\mathrm{X}$ with $\mathrm{RSH}$, and finally the reductive elimination of $\mathrm{Ar}-\mathrm{M}-\mathrm{SR}$ to form the desired $\mathrm{Ar}$ - SR coupling product. ${ }^{[4]}$ Interestingly, it has been found that the reactivity for the coupling of electron rich or poor aryl halides are quite different. In the presence of a strong base, the coupling of electron-poor aryl halides can be facilitated even in the absence of a transition metal catalyst under a comparatively high temperature $(>$ $\left.80{ }^{\circ} \mathrm{C}\right) .{ }^{[5]}$ For these electron-poor aryl halides, the reaction is assumed to proceed via $\mathrm{S}_{\mathrm{N}} \mathrm{Ar}$ mechanism. Despite significant efforts have been made in this transformation, it is surprising that so far most reported processes are mainly focused on the coupling of aryl halides and aryl thiols. ${ }^{[6]}$ However, the coupling of aryl halides with alkyl thiols is unfortunately still not well-established, even though alkyl aryl thioethers are also important synthetic intermediates. Based on this consideration, we were interested in investigating the $\mathrm{C}-\mathrm{S}$ cross-coupling of aryl halides with alkyl thiols under transition metal-free conditions. Surprisingly, it was found that the reaction could be smoothly proceeded even at room temperature. It is known that the $\mathrm{S}_{\mathrm{N}} \mathrm{Ar}$ reaction normally proceeds at high temperatures. Such a phenomenon motivated us to make further studies on the mechanism of this reaction. Therefore, we wish to report our recent findings on this work herein.

\section{Result and discussion}

The $\mathrm{C}-\mathrm{S}$ cross-coupling of electron-poor 4-bromoacetophenone (1a) and butanethiol (2a) was initially explored to examine the reactivity. Different solvents were

\footnotetext{
* Corresponding authors. E-mail: sunjing@lnpu.edu.cn, mingdong.zhou@lnpu.edu.cn

Received December 2, 2016; revised January 14, 2017; published online February 17, 2017

Project supported by the National Natural Science Foundation of China (No. 21101085), the Natural Science Foundation of Liaoning Province (No. 2015020196), the Fushun Science \& Technology Program (No. FSKJHT 201423), the Liaoning Excellent Talents Program in University (No. LJQ2012031), and the Talent Scientific Research Fund of Liaoning Shihua University (No. 2016XJJ-006).

国家自然科学基金(No. 21101085)、辽宁省自然科学基金(No. 2015020196)、抚顺市科技厅(No. FSKJHT 201423)、辽宁省高等学校优秀人才支持计划 (No. LJQ2012031)、辽宁石油化工大学引进人才科研启动基金(No. 2016XJJ-006)资助项目.
} 
firstly applied using 1.5 equiv. of $\mathrm{KO}^{t} \mathrm{Bu}$ at $100{ }^{\circ} \mathrm{C}$ under nitrogen protection. The reaction was not able to proceed in toluene, but moderate yield was achieved in tetrahydrofuran (THF) (Table 1, Entries 1 and 2). To our delight, excellent yield could be obtained when carrying out the reaction in pyridine, 1,2-dimethoxyethane (DME), dimethyl sulfoxide (DMSO) and $N, N$-dimethylformamide (DMF) (Entries $3 \sim$ 6). To our surprise, further studies indicated that excellent yields of 3aa could also be maintained when decreasing the temperature from $100{ }^{\circ} \mathrm{C}$ to room temperature (Entries $6 \sim$ 9 ). To further determine the reactivity, various bases were studied at room temperature. The results indicated that the replacement of $\mathrm{KO}^{t} \mathrm{Bu}$ with $\mathrm{NaO}^{t} \mathrm{Bu}, \mathrm{KOH}$ or $\mathrm{NaOH}$ also afforded excellent product yields at room temperature (Entries $10 \sim 12$ ), while $\mathrm{K}_{2} \mathrm{CO}_{3}$ led to a loweryield (Entry 13) and $\mathrm{Na}_{2} \mathrm{CO}_{3}$ proved to be not quite effective (Entry 14). Decreasing of the amount of $\mathrm{KO}^{t} \mathrm{Bu}$ to 1.0 equiv., the product yield also decreased (Entry 15).

Table $1 \mathrm{C}-\mathrm{S}$ cross-coupling of 4-bromoacetophenone and butanethiol $^{a}$

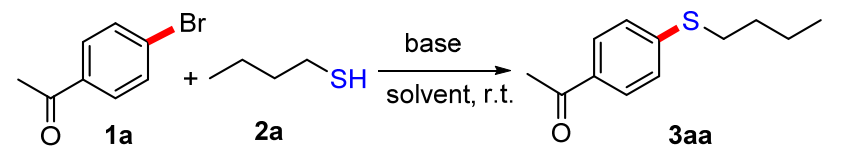

\begin{tabular}{clclc}
\hline Entry & Solvent & Temperature $/{ }^{\circ} \mathrm{C}$ & Base (equiv.) & Yield $^{b} \%$ \\
\hline 1 & Toluene & 100 & $\mathrm{KO}^{t} \mathrm{Bu}(1.5)$ & 0 \\
2 & THF & 100 & $\mathrm{KO}^{t} \mathrm{Bu}(1.5)$ & 52 \\
3 & Pyridine & 100 & $\mathrm{KO}^{t} \mathrm{Bu}(1.5)$ & $>99$ \\
4 & DME & 100 & $\mathrm{KO}^{t} \mathrm{Bu}(1.5)$ & $>99$ \\
5 & DMSO & 100 & $\mathrm{KO}^{t} \mathrm{Bu}(1.5)$ & $>99$ \\
6 & DMF & 100 & $\mathrm{KO}^{t} \mathrm{Bu}(1.5)$ & $>99$ \\
7 & DMF & 80 & $\mathrm{KO}^{t} \mathrm{Bu}(1.5)$ & $>99$ \\
8 & DMF & 50 & $\mathrm{KO}^{t} \mathrm{Bu}(1.5)$ & $>99$ \\
9 & DMF & r.t. & $\mathrm{KO}^{t} \mathrm{Bu}(1.5)$ & $>99\left(95^{c}\right)$ \\
10 & DMF & r.t. & $\mathrm{NaO}^{t} \mathrm{Bu}(1.5)$ & $>99$ \\
11 & DMF & r.t. & $\mathrm{NaOH}^{c}(1.5)$ & $>99$ \\
12 & DMF & r.t. & $\mathrm{KOH}(1.5)$ & $>99$ \\
13 & DMF & r.t. & $\mathrm{K}_{2} \mathrm{CO}_{3}(1.5)$ & 84 \\
14 & DMF & r.t. & $\mathrm{Na}_{2} \mathrm{CO}_{3}(1.5)$ & 8 \\
15 & DMF & r.t. & $\mathrm{KO}^{t} \mathrm{Bu}(1.0)$ & 83 \\
16 & DMF & r.t. & $\mathrm{KO}^{t} \mathrm{Bu}(2.0)$ & $>99$ \\
\hline
\end{tabular}

${ }^{a}$ Reaction conditions: 4-bromoacetophenone (1 mmol), butanethiol (1.1 mmol), base in $3 \mathrm{~mL}$ of solvent for $1 \mathrm{~h}$ under $\mathrm{N}_{2}$ protection; ${ }^{b} \mathrm{GC}$ yields; Isolated yields.

Based on the above studies, a condition A (1 mmol of 4-bromoacetophenone, $1.1 \mathrm{mmol}$ of butanethiol, 1.5 equiv. of $\mathrm{KO}^{t} \mathrm{Bu}$ in $3 \mathrm{~mL}$ of $\mathrm{DMF}$ at room temperature for $1 \mathrm{~h}$ under $\mathrm{N}_{2}$ protection was applied as a standard condition for further study on the substrate scope of this reaction (Table 2 ). It turned out that reactions between butanethiol and all examined electron-poor aryl bromides can be facilitated very well at room temperature, leading to the corresponding cross-coupling products in good yields. Excellent yields can be obtained for the coupling of 4-bromoacetophenone with heptanthiol, 2-methyl-2-propanethiol or cyclohexylthiol $(\mathbf{3 a a} \sim \mathbf{3 a d})$. A comparatively lower yield can be achieved when using bulkyl alkylthiols such as 3-methyl-2-butanethiol (3ae). For the sake of comparison, the coupling of 4-bromoacetophenone with aryl thiol such as 4-methylbenzenethiol and 4-chlorobenzenethiol was also conducted, and no obvious difference on activity was observed as compared to alkyl thiols (3af, 3ag). Additionally, excellent yields were also obtained for the coupling of various $\mathrm{CF}_{3}$ or $\mathrm{NO}_{2}$ substituted aryl bromide with butanethiol $(\mathbf{3 b a} \sim \mathbf{3 f a})$. However, this reaction did not occur when the substrates bearing with F, H or Me group (3ga 3ia). Obviously, the electronic effect of substituted aryl bromides had a significant impact on this transformation.

Table $2 \mathrm{C}-\mathrm{S}$ cross-coupling of aryl bromides with thiols ${ }^{a, b}$

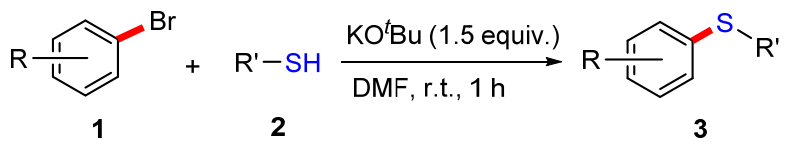<smiles>CCCCSc1ccc(C(C)=O)cc1</smiles>

3aa, $95 \%$<smiles>CCCCCCCSc1ccc(C(C)=O)cc1</smiles>

$3 a b, 94 \%$<smiles>CC(=O)c1ccc(Sc2ccc(C)cc2)cc1</smiles><smiles>CCCCSc1ccc(C(F)(F)F)cc1</smiles>

3ba, $93 \%$ 3ca, $95 \%$

3da, $95 \%$<smiles>CCCCSc1ccc(F)cc1</smiles>

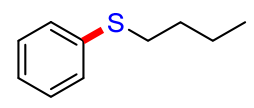

3ha, $0 \%$

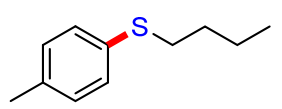

3ia, $0 \%$
${ }^{a}$ Reaction conditions: aryl halide $(1 \mathrm{mmol})$, thiol $(1.1 \mathrm{mmol}), \mathrm{KO}^{t} \mathrm{Bu}(1.5$ equiv.) in $3 \mathrm{~mL}$ of DMF at room temperature for $1 \mathrm{~h}$ under nitrogen atmosphere; ${ }^{b}$ Isolated yields.

\section{Mechanistic considerations}

As mentioned previously, the $\mathrm{S}_{\mathrm{N}} \mathrm{Ar}$ reaction normally occurs at high temperatures. In our case, the coupling reaction could be smoothly facilitated at room temperature. ${ }^{[5]}$ Additionally, Liu et $a l^{[5 \mathrm{~d}]}$ also found that the coupling of 
1-chloro-4-nitrobenzene with 1-octanethiol in the presence of 3 equiv. of KOH in PEG-600 (solvent) could proceed at room temperature, in which $90 \%$ of octyl $p$-nitrobenzyl thioether was obtained. Nevertheless, the mechanism was not discussed in this work. The main concern is whether $\mathrm{C}-\mathrm{S}$ coupling of electron-poor aryl halides with thiols can proceed via a $\mathrm{S}_{\mathrm{N}} \mathrm{Ar}$ mechanism at room temperature. Therefore, the mechanism needs to be carefully examined. First, if the reaction proceeds via an aryne mecha nism, two isomers (para and meta-substituted thioethers) should be observed in a roughly $1: 1$ molar ratio when using para-substituted aryl halides as substrates. ${ }^{[5 b, 7]}$ However, only one isomer could be detected for all examined electron-poor aryl bromides. Therefore, the aryne mechanism can be ruled out. A mechanism proceeding via a benzene radical intermediate induced by the $\mathrm{KO}^{t} \mathrm{Bu} / \mathrm{DMF}$ super-base system should not be ignored. $\mathrm{KO}^{t} \mathrm{Bu}$ or $\mathrm{NaO}^{t} \mathrm{Bu}$ has been found to be able to promote the direct $\mathrm{C}-\mathrm{H}$ arylation of unactivated benzene with aryl halides, which has been independently described by the group of Shi, Kong/Lei, and Shirakawa/Hayashi. ${ }^{[8 \sim 10]}$ Moreover, Yan et al. ${ }^{[11]}$ have also reported a series of $\mathrm{KO}^{t} \mathrm{Bu} / \mathrm{DMF}$ promoted radical coupling reactions. To further explore the mechanism, radical trapping experiments were carried out using $1 \sim 3$ equiv. of tetramethylpiperidine $N$-oxide (TEMPO) or 2,6-di-tert-butyl-4-methylphenol (BHT) as the radical scavengers in the presence of 1.5 equiv. of $\mathrm{KO}^{t} \mathrm{Bu}$ in $\mathrm{DMF}$ at room temperature. However, the reaction could not be significantly hindered by both scavengers. Thus, a benzene radical based mechanism appears unlikely. Therefore, the $\mathrm{C}-\mathrm{S}$ coupling using electron-poor aryl halides might proceed via a $S_{N} A r$ mechanism, even at room temperature, which is in accordance to the reported mechanism. ${ }^{[5 b]}$

\section{Conclusions}

$\mathrm{C}-\mathrm{S}$ cross-coupling of aryl bromides with alkyl thiols under transition metal-free conditions is described. The examined coupling of electron-poor aryl halides with alkylthiols proved to be highly effective at room temperature under transition metal-free conditions. The reaction is applicable for aryl bromides bearing various electron-withdrawing groups. The reaction is also applicable for various linear, bulkyl and cyclic alkyl thiols. Reaction mechanism studies indicate that the reaction likely undergoes a $\mathrm{S}_{\mathrm{N}} \mathrm{Ar}$ mechanism, even at room temperature. This reaction protocol provides an efficient and cost-effective method for the synthesis of alkyl aryl thioethers under mild conditions.

\section{Experimental}

\subsection{General method}

All reactions were carried out under a nitrogen atmosphere with standard Schlenk techniques. All solvents were purified and dried according to standard methods prior to use. The GC-MS spectra were determined by a Thermo Fisher ISQ GC-MS; NMR spectra were recorded on a Bruker Ascend HD 500 spectrometer; IR spectra were rec- orded on a Nicolet 6700 FT-IR spectrometer; The melting points of compounds were obtained on a Shang Hai Shen Guang WRS-3 melting point instrument; HRMS (ESI) determinations were carried out on a Bruker Daltonics McriOTOF II spectrometer.

\subsection{General procedure for the coupling reaction of aryl bromides with thiols}

In a typical procedure, aryl bromide $(1.0 \mathrm{mmol})$, thiols $(1.1 \mathrm{mmol})$ and $\mathrm{KO}^{t} \mathrm{Bu}(168 \mathrm{mg}, 1.5 \mathrm{mmol})$ were added to a Schlenk tube under an atmosphere of nitrogen. DMF (3 mL) was then injected under vigorous stirring $1 \mathrm{~h}$. After the reaction, the mixture was quenched by addition of distilled water. The organic phase was then separated, and the aqueous phase was extracted with ethyl acetate for three times. The combined organic layers were dried with $\mathrm{MgSO}_{4}$, and the sample was conducted for GC or GC-MS analysis. The sample solution was further evaporated under vacuum to remove the solvent. The obtained crude product was purified by column chromatography (eluent, petroleum) on silica gel to afford the desired thioethers.

1-(4-(Butylthio)phenyl)ethanone (3aa): ${ }^{[12]}$ Colorless liquid, $198 \mathrm{mg}, 95 \%$ yield. ${ }^{1} \mathrm{H} \mathrm{NMR}\left(500 \mathrm{MHz}, \mathrm{CDCl}_{3}\right) \delta$ : 7.85 (d, $J=8.5 \mathrm{~Hz}, 2 \mathrm{H}), 7.29$ (d, $J=8.5 \mathrm{~Hz}, 2 \mathrm{H}), 2.98$ (t, $J=7.0 \mathrm{~Hz}, 2 \mathrm{H}), 2.55(\mathrm{~s}, 3 \mathrm{H}) 1.71 \sim 1.65(\mathrm{~m}, 2 \mathrm{H}), 1.51 \sim$ $1.44(\mathrm{~m}, 2 \mathrm{H}), 0.94(\mathrm{t}, J=7.0 \mathrm{~Hz}, 3 \mathrm{H})$.

1-(4-(Heptylthio)phenyl)ethanone (3ab): ${ }^{[13]}$ Colorless liquid, $207 \mathrm{mg}, 94 \%$ yield. ${ }^{1} \mathrm{H}$ NMR $\left(500 \mathrm{MHz} \mathrm{CDCl}_{3}\right) \delta$ : $7.86(\mathrm{~d}, J=8.5 \mathrm{~Hz}, 2 \mathrm{H}), 7.30$ (d, $J=9.0 \mathrm{~Hz}, 2 \mathrm{H}), 2.99$ (t, $J=7.5 \mathrm{~Hz}, 2 \mathrm{H}), 2.57(\mathrm{~s}, 3 \mathrm{H}), 1.73 \sim 1.66(\mathrm{~m}, 2 \mathrm{H}), 1.46 \sim$ $1.43(\mathrm{~m}, 2 \mathrm{H}), 1.34 \sim 1.26(\mathrm{~m}, 6 \mathrm{H}), 0.89(\mathrm{t}, J=7.0 \mathrm{~Hz}, 3 \mathrm{H})$.

1-(4-((3-Methylbutan-2-yl)thio)phenyl)ethanone (3ac): Colorless liquid, $162 \mathrm{mg}, 84 \%$ yield. ${ }^{1} \mathrm{H}$ NMR $(500 \mathrm{MHz}$, $\left.\mathrm{CDCl}_{3}\right) \delta: 7.85(\mathrm{~d}, J=8.5 \mathrm{~Hz}, 2 \mathrm{H}), 7.35(\mathrm{~d}, J=8.5 \mathrm{~Hz}, 2 \mathrm{H})$, $3.39 \sim 3.34(\mathrm{~m}, 1 \mathrm{H}), 2.55(\mathrm{~s}, 3 \mathrm{H}), 1.98 \sim 1.92(\mathrm{~m}, 1 \mathrm{H}), 1.30$ $(\mathrm{d}, J=7.0 \mathrm{~Hz}, 3 \mathrm{H}), 1.04 \sim 1.00(\mathrm{~m}, 6 \mathrm{H}) ;{ }^{13} \mathrm{C}$ NMR $(125$ $\left.\mathrm{MHz}, \mathrm{CDCl}_{3}\right) \delta: 196.9,144.3,134.0,128.6,128.1,48.1$, $32.5,26.3,19.9,18.4,16.9$; IR (neat) $v: 2966,2940,2874$, 1680, 1589, 1552, 1394, 1350, 1262, 1181, 1097, 957, 817, $762,601 \mathrm{~cm}^{-1}$; HRMS (EI) calcd for $\mathrm{C}_{13} \mathrm{H}_{18} \mathrm{SO}: 222.1078$, found 222.1080

1-(4-(Cyclohexylthio)phenyl)ethanone (3ad) $:^{[4 e, 14]}$ White solid, $161 \mathrm{mg}, 78 \%$ yield. m.p. $67.4 \sim 67.6{ }^{\circ} \mathrm{C}$ (lit. ${ }^{[14]} 66 \sim$ $\left.68{ }^{\circ} \mathrm{C}\right) ;{ }^{1} \mathrm{H}$ NMR $\left(500 \mathrm{MHz}, \mathrm{CDCl}_{3}\right) \delta: 7.85(\mathrm{~d}, J=8.5 \mathrm{~Hz}$, $2 \mathrm{H}), 7.35(\mathrm{~d}, J=8.5 \mathrm{~Hz}, 2 \mathrm{H}), 3.33 \sim 3.30(\mathrm{~m}, 1 \mathrm{H}), 2.56(\mathrm{~s}$, $3 \mathrm{H}), 2.09 \sim 1.95(\mathrm{~m}, 4 \mathrm{H}), 1.80 \sim 1.77(\mathrm{~m}, 4 \mathrm{H}), 1.66 \sim 1.59$ $(\mathrm{m}, 2 \mathrm{H})$.

1-(4-(tert-Butylthio)phenyl)ethanone (3ae) $:^{[15]}$ Colorless liquid, $124 \mathrm{mg}, 69 \%$ yield. ${ }^{1} \mathrm{H}$ NMR $\left(500 \mathrm{MHz} \mathrm{CDCl}_{3}\right) \delta$ : $7.91(\mathrm{~d}, J=8.5 \mathrm{~Hz}, 2 \mathrm{H}), 7.62$ (d, $J=8.5 \mathrm{~Hz}, 2 \mathrm{H}), 2.60$ (s, $3 \mathrm{H}), 1.32(\mathrm{~s}, 9 \mathrm{H})$.

1-(4-(p-Tolylthio)phenyl)ethanone (3af): ${ }^{[6 \mathrm{~b}]}$ Yellow solid, $197 \mathrm{mg}, 92 \%$ yield. m.p. 94.1 94.2 ${ }^{\circ} \mathrm{C}$ (lit. ${ }^{[6 \mathrm{~b}]} 89 \sim$ $\left.92{ }^{\circ} \mathrm{C}\right) ;{ }^{1} \mathrm{H}$ NMR $\left(500 \mathrm{MHz}, \mathrm{CDCl}_{3}\right) \delta: 7.83 \sim 7.79(\mathrm{~m}, 2 \mathrm{H})$, 7.42 (d, $J=8.5 \mathrm{~Hz}, 2 \mathrm{H}), 7.23(\mathrm{~d}, J=8.0 \mathrm{~Hz}, 2 \mathrm{H}), 7.16$ (d, $J=8.0 \mathrm{~Hz}, 2 \mathrm{H}), 2.54$ (s, 3H), 2.39 (s, 3H).

1-(4-((4-Chlorophenyl)thio)phenyl)ethanone (3ag): ${ }^{[16]}$ 
White solid, $212 \mathrm{mg}, 91 \%$ yield. m.p. $56.0 \sim 56.7{ }^{\circ} \mathrm{C}$ (lit. $\left.{ }^{[16]} 40 \sim 42{ }^{\circ} \mathrm{C}\right) ;{ }^{1} \mathrm{H}$ NMR $\left(500 \mathrm{MHz}, \mathrm{CDCl}_{3}\right) \delta: 7.84(\mathrm{~d}$, $J=9.0 \mathrm{~Hz}, 2 \mathrm{H}), 7.41 \sim 7.35(\mathrm{~m}, 4 \mathrm{H}), 7.22(\mathrm{~d}, J=8.5 \mathrm{~Hz}$, 2H), 2.55 (s, 3H).

Butyl(2-(trifluoromethyl)phenyl)sulfane (3ba): Colorless liquid, $217 \mathrm{mg}$, 93\% yield. ${ }^{1} \mathrm{H} \mathrm{NMR}\left(500 \mathrm{MHz}, \mathrm{CDCl}_{3}\right) \delta$ : $7.63(\mathrm{~d}, J=7.5 \mathrm{~Hz}, 1 \mathrm{H}), 7.46 \sim 7.44(\mathrm{~m}, 2 \mathrm{H}), 7.25 \sim 7.23$ $(\mathrm{m}, 1 \mathrm{H}), 2.97(\mathrm{t}, J=7.5 \mathrm{~Hz}, 2 \mathrm{H}), 1.69 \sim 1.63(\mathrm{~m}, 2 \mathrm{H})$, $1.49 \sim 1.41(\mathrm{~m}, 2 \mathrm{H}), 0.93(\mathrm{t}, J=9.0 \mathrm{~Hz}, 3 \mathrm{H}) ;{ }^{13} \mathrm{C} \mathrm{NMR}(125$ $\left.\mathrm{MHz}, \mathrm{CDCl}_{3}\right) \delta: 137.1,131.8,129.9,126.7,125.2,33.6$, $30.8,21.9,13.5$; IR (neat) $v: 2959,2931,2869,1596,1568$, 1471, 1433, 1310, 1255, 1171, 1126, 1023, $760 \mathrm{~cm}^{-1}$; HRMS (EI) calcd for $\mathrm{C}_{11} \mathrm{H}_{13} \mathrm{~F}_{3} \mathrm{~S}$ : 234.0690, found 234.0683.

Butyl(3-(trifluoromethyl)phenyl)sulfane (3ca): ${ }^{[17]}$ Colorless liquid, $223 \mathrm{mg}, 95 \%$ yield. ${ }^{1} \mathrm{H}$ NMR $(500 \mathrm{MHz}$, $\left.\mathrm{CDCl}_{3}\right) \delta: 7.52(\mathrm{~s}, 1 \mathrm{H}), 7.47 \sim 7.45(\mathrm{~m}, 1 \mathrm{H}), 7.39 \sim 7.37(\mathrm{~m}$, 2H), $2.96(\mathrm{t}, J=9.5 \mathrm{~Hz}, 2 \mathrm{H}), 1.69 \sim 1.60(\mathrm{~m}, 2 \mathrm{H}), 1.49 \sim$ $1.41(\mathrm{~m}, 2 \mathrm{H}), 0.94(\mathrm{t}, J=9.5 \mathrm{~Hz}, 3 \mathrm{H})$.

Butyl(4-(trifluoromethyl)phenyl)sulfane (3da): ${ }^{[18]}$ Colorless liquid, $223 \mathrm{mg}, 95 \%$ yield. ${ }^{1} \mathrm{H}$ NMR $(500 \mathrm{MHz}$, $\left.\mathrm{CDCl}_{3}\right) \delta: 7.50(\mathrm{~d}, J=8.5 \mathrm{~Hz}, 2 \mathrm{H}), 7.34(\mathrm{~d}, J=8.5 \mathrm{~Hz}, 2 \mathrm{H})$, $2.96(\mathrm{t}, J=7.0 \mathrm{~Hz}, 2 \mathrm{H}), 1.69 \sim 1.63(\mathrm{~m}, 2 \mathrm{H}), 1.51 \sim 1.45$ (m, 2H), 0.94 (t, $J=7.5 \mathrm{~Hz}, 3 \mathrm{H})$.

Butyl(2-nitrophenyl)sulfane (3ea): ${ }^{[12]}$ Colorless liquid, $192 \mathrm{mg}, 91 \%$ yield. ${ }^{1} \mathrm{H}$ NMR $\left(500 \mathrm{MHz}, \mathrm{CDCl}_{3}\right) \delta: 8.17 \sim$ $8.15(\mathrm{~m}, 1 \mathrm{H}), 7.54 \sim 7.51(\mathrm{~m}, 1 \mathrm{H}), 7.41(\mathrm{~d}, J=8.0 \mathrm{~Hz}, 1 \mathrm{H})$, $7.22 \sim 7.19(\mathrm{~m}, 1 \mathrm{H}), 2.94(\mathrm{t}, J=7.5 \mathrm{~Hz}, 2 \mathrm{H}), 1.72 \sim 1.67$ (m, 2H), $1.53 \sim 1.47(\mathrm{~m}, 2 \mathrm{H}), 0.95(\mathrm{t}, J=7.5 \mathrm{~Hz}, 3 \mathrm{H})$.

Butyl(4-nitrophenyl)sulfane (3fa): ${ }^{[12]}$ Colorless liquid, $194 \mathrm{mg}, 92 \%$ yield. ${ }^{1} \mathrm{H}$ NMR $\left(500 \mathrm{MHz}, \mathrm{CDCl}_{3}\right) \delta: 8.11$ (d, $J=9.0 \mathrm{~Hz}, 2 \mathrm{H}), 7.31(\mathrm{~d}, J=9.0 \mathrm{~Hz}, 2 \mathrm{H}), 3.01(\mathrm{t}, J=8.0 \mathrm{~Hz}$, $2 \mathrm{H}), 1.73 \sim 1.66(\mathrm{~m}, 2 \mathrm{H}), 1.53 \sim 1.47(\mathrm{~m}, 2 \mathrm{H}), 0.96(\mathrm{t}, J=$ $7.5 \mathrm{~Hz}, 3 \mathrm{H})$.

Supporting Information ${ }^{1} \mathrm{H}$ NMR and ${ }^{13} \mathrm{C}$ NMR spectra. The Supporting Information is available free of charge via the Internet at http://sioc-journal.cn/.

\section{References}

[1] (a) Yao, H.; Richardson, D. E. J. Am. Chem. Soc. 2003, 125, 6211. (b) Qin, Z.; Kastrati, I.; Chandrasena, R. E. P.; Liu, H.; Yao, P.; Petukhov, P. A.; Bolton, J. L.; Thatcher, G. R. J. J. Med. Chem. 2007 50, 2682.

(c) Bagley, M. C.; Davis, T.; Dix, M. C.; Rokicki, M.; Kipling, D. Bioorg. Med. Chem. Lett. 2007, 17, 5107.

(d) Bryan, C. S.; Braunger, J. A.; Lautens, M. Angew. Chem. Int. Ed. 2009, 121, 7198 .

(e) Beletskaya, I. P.; Ananikov, V. P. Chem. Rev. 2011, 111, 1596.

(f) Ubale, A. U.; Bhute, M. V.; Malpe, G. P.; Raut, P. P.; Chipade, K. S.; Ibrahim, S. G. J. Sadui Chem. Soc. 2016, 20, 227.

(g) Zhou, Y. B.; Gao, F.; Zhao, Y.; Lu, J. J. Sadui Chem. Soc. 2014, $18,589$.

(h) Zaafarany, I.; Boller, H. J. Sadui Chem. Soc. 2010, 14, 183.

(i) An, Y. N.; Li, J. X.; Li, M..; Li, C. S.; Yang, S. R. Chin. J. Org. Chem. 2017, 37, 720 (in Chinese).

(安艳妮, 李建晓, 李蒙, 李春生, 杨少容, 有机化学, 2017, 37, 720.)
[2] (a) Byeun, A.; Baek, K.; Han, M. S.; Lee, S. Tetrahedron Lett. 2013, 54, 6712 .

(b) Alvaroa, E.; Hartwig, J. F. J. Am. Chem. Soc. 2009, 131, 7858.

(c) Li, J. X.; Li, C. S.; Yang, S. R.; An, Y. N.; Wu, W. Q.; Jiang, H. F. J. Org. Chem. 2016, 81, 2875 .

(d) Li, J. X.; Li, C. S.; Yang, S. R.; An, Y. N.; Wu, W. Q.; Jiang, H. F. J. Org. Chem. 2016, 81, 7771.

[3] (a) Luo, F.; Pan, C.; Li, L. P.; Chen, F.; Cheng, J. Chem. Commun. 2011, 47, 5304.

(b) Mallick, S.; Rana, S.; Parida, K. Dalton. Trans. 2011, 40, 9169.

[4] (a) Zhang, J.; Medley, C. M.; Krause, J. A.; Guan, H. Organometallics 2010, 29, 6393.

(b) Venkanna, G. T.; Arman, H. D.; Tonzetich, Z. J. ACS Catal. 2014, 4, 2941.

(c) Jammi, S.; Barua, P.; Rout, L.; Saha, P.; Punniyamurthy, T. Tetrahedron Lett. 2008, 49, 1484.

(d) Baldovino-Pantaleón, O.; Hernández-Ortega, S.; Morales-Morales, D. Adv. Syn. Catal. 2006, 348, 236.

(e) Guan, P.; Cao, C.; Liu, Y.; Li, Y.; He, P.; Chen, Q.; Liu, G.; Shi, Y. Tetrahedron Lett. 2012, 53, 5987.

[5] (a) Zhang, Y. G.; Ngeow, K. C.; Ying, J. Y. Org. Lett. 2007, 9, 3495. (b) Yuan, Y.; Thomé, I.; Kim, S. H.; Chen, D.; Beyer, A.; Bonnamour, J.; Zuidema, E.; Chang, S.; Bolm, C. Adv. Synth. Catal. 2010, 352, 2892.

(c) Cano, R.; Ramón, D. J.; Yus, M. J. Org. Chem. 2011, 76, 654.

(d) Duan, Z.; Ranjit, S.; Liu, X. Org. Lett. 2010, 12, 2430.

[6] (a) Shi, Y.; Cai, Z.; Guan, P.; Pang, G. Synth. Let. 2011, 22, 2090.

(b) Fu, C. F.; Liu, Y. H.; Peng, S. M.; Liu, S. T. Tetrahedron 2010, 66, 2119.

(c) Ma, D.; Geng, Q.; Zhang, H.; Jiang, Y. Angew. Chem., Int. Ed. 2010, 49, 1291.

(d) Xu, R.; Wan, J. P.; Mao, H.; Pan, Y. J. Am. Chem. Soc. 2010, 132, 15531 .

(e) Wu, J. R.; Lin, C. H.; Lee, C. F. Chem. Commun. 2009, 29, 4450 .

(f) Iglesias, M. J.; Prieto, A.; Nicasio, M. C. Adv. Synth. Catal. 2010, 352, 949.

[7] (a) Bradshaw, J. S.; Chen, E. Y.; Hales, R. H.; South, J. A. J. Org. Chem. 1972, 37, 2051.

(b) March, J. Advanced Organic Chemistry, Vol. 4, John Wiley \& Sons, Inc, New York, 1985, p. 576.

(c) Varala, R.; Ramu, E.; Alam, M. M.; Adapa, S. R. Chem. Lett. 2004, 33, 1614.

[8] Sun, C.; Li, H.; Yu, D.; Yu, M.; Zhou, X.; Lu, X.; Huang, K.; Zheng, S.; Li, B.; Shi, Z. Nat. Chem. 2010, 2, 044.

[9] Liu, W.; Cao, H.; Zhang, H.; Zhang, H.; Chung, K. H.; He, C.; Wang, H.; Kong, F. Y.; Lei, A. J. Am. Chem. Soc. 2010, 132, 16737.

[10] Shirakawa, E.; Itoh, K.; Higashino, T.; Hayashi, T. J. Am. Chem. Soc. 2010, 132, 15537.

[11] (a) Wei, W. T.; Liu, Y.; Ye, L. M.; Lei, R. H.; Zhang, X. J.; Yan, M. Org. Biomol. Chem. 2015, 13, 817.

(b) Chen, Y. Y.; Zhang, N. N.; Ye, L. M.; Chen, J. H.; Shun, X.; Zhang, X. J.; Yan, M. RSC Adv. 2015, 5, 48046.

[12] Qiao, Z.; Wei, J.; Jiang, X. Org. Lett. 2014, 16, 1212.

[13] Xu, J. A.; Wei, T. Z.; Xia, J. K.; Zhang, Q. H.; Wu, H. S. Chirality 2004, 16, 341.

[14] Zhang, H.; Cao, W. G.; Ma, D. W. Synth. Commun. 2007, 37, 25.

[15] Martyn, J.; Soren, L. B.; Mogens, B. N. J. Org. Chem. 2013, 78, 4348.

[16] Gorczynski, M. J.; Leal, R. M.; Mooberry, S. L.; Bushweller, J. H.; Brown, M. L. Bioorg. Med. Chem. 2004, 12, 1029.

[17] Jean, M.; Renault, J.; Weghe, P. V. D.; Asao, N. Tetrahedron Lett. 2010, $51,378$.

[18] Taniguchi, N.; Onami, T. J. Org. Chem. 2004, 69, 915.

(Li, L.; Lu, Z.) 